\title{
Seismo-acoustic ray model benchmarking against experimental tank data
}

\author{
Orlando Camargo Rodríguez ${ }^{\text {a) }}$ \\ LARSYS, Campus de Gambelas, Universidade do Algarve, PT-8005-139 Faro, Portugal \\ Jon M. Collis \\ Department of Applied Mathematics and Statistics, Colorado School of Mines, 1500 Illinois Street, Golden, \\ Colorado 80401 \\ Harry J. Simpson \\ Physical Acoustic Branch Code 7136, Naval Research Laboratory, 4555 Overlook Avenue Southwest, \\ Washington, DC 20375 \\ Emanuel Ey, Joseph Schneiderwind, and Paulo Felisberto \\ LARSyS, Campus de Gambelas, Universidade do Algarve, PT-8005-139 Faro, Portugal
}

(Received 1 February 2012; revised 18 June 2012; accepted 21 June 2012)

\begin{abstract}
Acoustic predictions of the recently developed TRACEO ray model, which accounts for bottom shear properties, are benchmarked against tank experimental data from the EPEE-1 and EPEE-2 (Elastic Parabolic Equation Experiment) experiments. Both experiments are representative of signal propagation in a Pekeris-like shallow-water waveguide over a non-flat isotropic elastic bottom, where significant interaction of the signal with the bottom can be expected. The benchmarks show, in particular, that the ray model can be as accurate as a parabolic approximation model benchmarked in similar conditions. The results of benchmarking are important, on one side, as a preliminary experimental validation of the model and, on the other side, demonstrates the reliability of the ray approach for seismo-acoustic applications.
\end{abstract}

(C) 2012 Acoustical Society of America. [http://dx.doi.org/10.1121/1.4734236]

PACS number(s): 43.30.Zk, 43.30.Ma, 43.20.Dk [AIT]

Pages: $709-717$

\section{INTRODUCTION}

Tank experiments constitute a fundamental reference for underwater acoustic modeling, by providing valuable data for model benchmarking. In particular these types of experiments are important because of the difficulties and costs involved with obtaining high-quality ocean acoustic data at sea. In contrast with benchmarking analytic solutions, ${ }^{1-5}$ which are generally difficult to obtain for a broad set of geometries and boundary properties, benchmarking to tank experimental data imposes important constraints to numerical models. On one side, propagation conditions can be carefully controlled; on the other side, despite such control, mismatch will be always observed since the numerical model is an approximation of the theoretical solution. The importance of benchmarking to tank experimental data was shown, for example, by the EPEE1 (Ref. 6) and EPEE-2 (Elastic Parabolic Equation Experiment) (Ref. 7) experiments, which demonstrated the excellent accuracy of the ROTVARS model, based on the variable rotated elastic parabolic equation. ${ }^{8}$ The high quality of the data acquired during these tank experiments is extremely important because both experiments are representative of propagation in a shallow water waveguide, with an elastic bottom and rangedependent bathymetry involving sharp slope changes. Ray models $^{9-12}$ are also interesting candidates for benchmarking against the tank experimental data. The ray solution to the

\footnotetext{
a) Author to whom correspondence should be addressed. Electronic mail: orodrig@ualg.pt
}

acoustic wave equation is an asymptotic approximation, which improves as frequency increases, and ray methods are computationally efficient in waveguides with complex characteristics, such as variable boundaries and range-independent or range-dependent sound speed distributions. Additionally, for a ray model to be accurate under such conditions, shear effects need to be included as well. Naturally, a question arises whether a ray model will be able to exhibit the same degree of accuracy as a parabolic equation solution, when benchmarked (in particular) to the data of the EPEE-1 and EPEE-2 tank experiments. The main purpose of the discussion presented here is to develop a systematic benchmarking of a ray model against such experimental data. To this end the tank experiments are briefly reviewed in Sec. II, while Sec. III describes the recently developed TRACEO ray model, which is benchmarked in detail in Sec. IV. The conclusions of benchmarking and future work are presented in Sec. V.

\section{THE EPEE-1 AND EPEE-2 TANK EXPERIMENTS}

The tank experiments are described in great detail in the literature ${ }^{6,7}$ therefore, a sufficiently compact description is presented in this section. Polyvinyl chloride (PVC) slabs with the elastic parameters given in Table I were suspended in a water tank by cables, that were attached to each slab corner at substantial distances from the sound source to avoid reflections. Source and receiver hydrophones were positioned over the slabs with a robotic arm, allowing for accurate positioning. Sound speed in the water is considered constant and corresponds to $1482 \mathrm{~m} / \mathrm{s}$. Acoustic 
TABLE I. PVC elastic properties at $300 \mathrm{kHz}(\lambda$ represents the acoustic wavelength).

\begin{tabular}{lcc}
\hline \hline Parameter & Units $^{\mathrm{a}}$ & Value \\
\hline Density & $\mathrm{kg} / \mathrm{m}^{3}$ & 1378 \\
Compressional speed & $\mathrm{m} / \mathrm{s}$ & 2290 \\
Shear speed & $\mathrm{m} / \mathrm{s}$ & 1050 \\
Compressional attenuation & $\mathrm{dB} / \lambda$ & 0.76 \\
Shear attenuation & $\mathrm{dB} / \lambda$ & 1.05 \\
\hline \hline
\end{tabular}

${ }^{\mathrm{a}} \mathrm{A}$ note of advice: Compressional and shear attenuations are given in Ref. 6 as $0.33 \mathrm{~dB} / \mathrm{m}$ and $1.00 \mathrm{~dB} / \mathrm{m}$, respectively. Attenuation is given here in $\mathrm{dB} / \lambda$, which are the units used by the ROTVARs model.

transmissions were performed for a wide set of frequencies, up to $1 \mathrm{MHz}$, but for the purposes of benchmarking only three frequencies are here considered, namely, 125, 200, and $275 \mathrm{kHz}$. Due to the nature of the source used in the experiment, data within the $100-300 \mathrm{kHz}$ band are considered valid for comparison purposes.

Acoustic propagation calculations are performed at a scale of 1000:1; thus, for a proper modification of parameter values the following conversion of units is adopted: experimental frequencies in $\mathrm{kHz}$ become model frequencies in $\mathrm{Hz}$ and experimental lengths in mm become model lengths in $\mathrm{m}$ (for instance, an experimental frequency of $100 \mathrm{kHz}$ becomes a model frequency of $100 \mathrm{~Hz}$ and an experimental distance of $10 \mathrm{~mm}$ becomes a model distance of $10 \mathrm{~m}$ ). Sound speeds remain unchanged, as well as compressional and shear attenuations, which are given in $\mathrm{dB} / \lambda$ (where $\lambda$ stands for the acoustic wavelength). In EPEE-1 the slab allowed both range-independent, "flat," and range-dependent

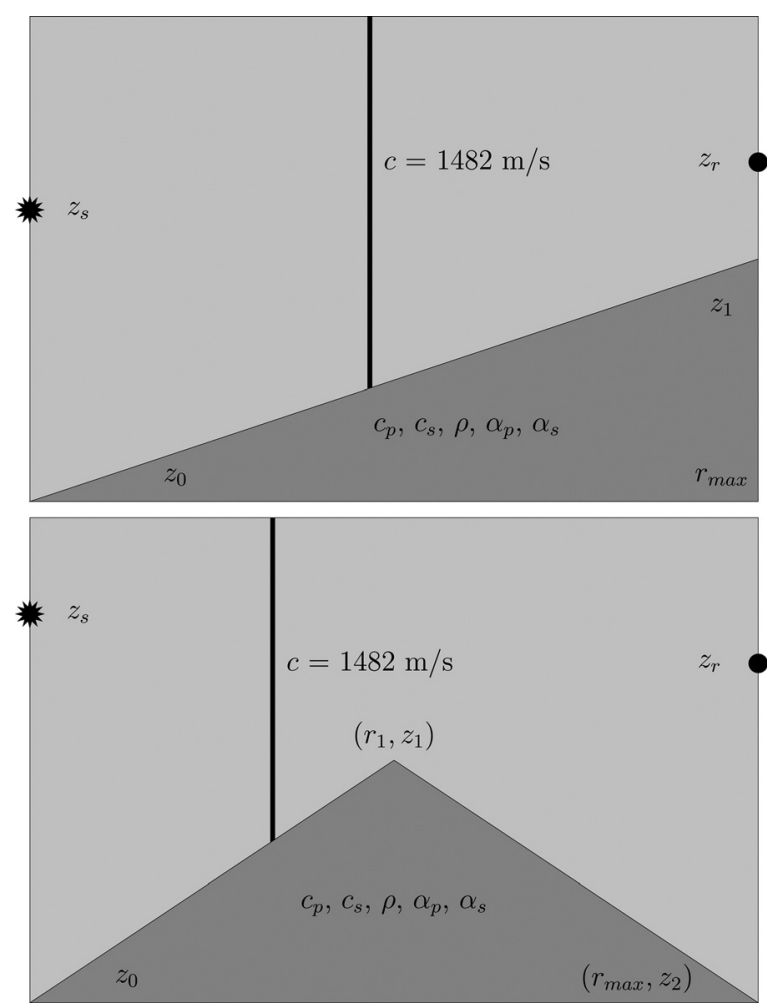

FIG. 1. EPEE-1, sloped case (top) and EPEE-2, upslope to downslope case (bottom). waveguides (see Fig. 1). In EPEE-2 the slab geometry allowed three different types of range-dependent bottom bathymetries, namely, flat to downslope, upslope to flat, and upslope to downslope. Different configurations of the acoustic source and receiver were considered in both experiments, but the benchmarking presented here will be limited to a single position of both source and receiver; geometric parameters for the waveguides of both experiments are shown in Table II.

\section{THE RAY MODEL}

The ray model benchmarked in the current work is the TRACEO Gaussian beam model, which is under current development at the SiPLAB of the University of Algarve. ${ }^{13}$ The code TRACEO was developed in order to

(1) Predict acoustic pressure and particle velocity in environments with elaborate upper and lower boundaries, which can be characterized by range-dependent compressional and shear properties. Modeling particle velocity is important for vector sensor applications and can be used, in particular, for geoacoustic inversion with high frequency data. ${ }^{14-16}$

(2) Include one or more targets in the waveguide.

(3) Produce ray, eigenray, amplitude, and travel time information. In particular, eigenrays are to be calculated even if rays are reflected backwards on targets located beyond the current position of the hydrophone.

The following sections compactly describe the theory behind TRACEO calculations of acoustic pressure, shear inclusion and particle velocity calculations; a numerical example is presented as well.

\section{A. Theoretical background}

The starting point for the general description of a threedimensional Gaussian beam is given by the expression ${ }^{17}$

$$
P(s, \boldsymbol{n})=\frac{1}{4 \pi} \sqrt{\frac{c(s)}{c(0)} \frac{\cos \theta(0)}{\operatorname{det} \boldsymbol{Q}}} \exp \left\{-i \omega\left[\tau(s)+\frac{1}{2}(\boldsymbol{M} n \cdot \boldsymbol{n})\right]\right\}
$$

where $s$ stands for the ray arclength, $\boldsymbol{n}$ is the normal to the ray (such normal lies on a plane, which will be introduced later), $\boldsymbol{M}$ and $\boldsymbol{Q}$ are $2 \times 2$ matrices (whose meaning will be explained below), the center dot represents the inner vector

TABLE II. Geometric parameters for the waveguides of the EPEE-1 and EPEE-2 tank experiments.

\begin{tabular}{lccccc}
\hline \hline & Flat & Upslope & Flat/down & Up/flat & Up/down \\
\hline$z_{s}(\mathrm{~m})$ & 69.1 & 63.4 & 74.2 & 73.5 & 72.1 \\
$z_{r}(\mathrm{~m})$ & 137.1 & 15.6 & 75.8 & 78.7 & 74.8 \\
$z_{0}(\mathrm{~m})$ & 144.7 & 132.9 & 143.9 & 245.9 & 183.0 \\
$z_{1}(\mathrm{~m})$ & 145.4 & 45.4 & 152.1 & 151.2 & 147.2 \\
$z_{2}(\mathrm{~m})$ & N/A & N/A & 238.2 & 145.7 & 198.4 \\
$r_{1}(\mathrm{~m})$ & N/A & N/A & 988.8 & 985.9 & 1000.3 \\
$r_{\max }(\mathrm{m})$ & 1200 & 1200 & 1898.0 & 1898.0 & 1898.0 \\
\hline
\end{tabular}


product, $\theta(0)$ is the initial ray elevation (i.e., the angle relative to the horizontal plane, which is formed by the $X$ and $Y$ axes; the angle on the $X Y$ plane relative to the $X$ axis will be called, hereafter, the azimuth), and $c(s)$ represents the sound speed along the ray trajectory:

$$
c(s)=c(s, 0) \text {. }
$$

The travel time $\tau(s)$ in Eq. (1) is calculated solving the set of Eikonal equations ${ }^{18}$

$$
\begin{aligned}
& \frac{d x}{d s}=c(s) \sigma_{x}, \quad \frac{d y}{d s}=c(s) \sigma_{y}, \quad \frac{d z}{d s}=c(s) \sigma_{z}, \\
& \frac{d \sigma_{x}}{d s}=-\frac{1}{c^{2}} \frac{\partial c}{\partial x}, \quad \frac{d \sigma_{y}}{d s}=-\frac{1}{c^{2}} \frac{\partial c}{\partial y}, \quad \frac{d \sigma_{z}}{d s}=-\frac{1}{c^{2}} \frac{\partial c}{\partial z},
\end{aligned}
$$

where $\sigma_{x}, \sigma_{y}$, and $\sigma_{z}$ stand for the components of the vector of sound slowness. The derivatives $d x / d s, d y / d s$, and $d z / d s$ define the ray tangent $\boldsymbol{e}_{s}$; the plane perpendicular to $\boldsymbol{e}_{s}$ defines the plane normal to the ray. Introducing on such plane a pair of unitary and orthogonal vectors $\boldsymbol{e}_{1}$ and $\boldsymbol{e}_{2}$ one can write the ray normal as

$$
\boldsymbol{n}=n_{1} \boldsymbol{e}_{1}+n_{2} \boldsymbol{e}_{2}
$$

where $n_{1}$ and $n_{2}$ are arbitrary quantities. The matrices $\boldsymbol{M}$ and $Q$ are required to be complex; therefore, the imaginary part of the product $\boldsymbol{M} n \cdot \boldsymbol{n}$ induces a Gaussian decay of beam amplitude along $\boldsymbol{n}$, while the real part introduces phase corrections to the travel time. As long as $\operatorname{det} Q \neq 0$ the solution given by Eq. (1) does not exhibit singularities. Besides $\boldsymbol{Q}$ and $\boldsymbol{M}$ the Gaussian beam approximation involves two additional $2 \times 2$ matrices, represented generally as $\boldsymbol{P}$ and $\boldsymbol{C}$; all four matrices are related through the following relationships: ${ }^{19}$

$$
\begin{aligned}
& \boldsymbol{M}=\boldsymbol{P} \boldsymbol{Q}^{-1}, \\
& \frac{d}{d s} \boldsymbol{Q}=c(s) \boldsymbol{P}, \quad \frac{d}{d s} \boldsymbol{P}=-\frac{1}{c^{2}(s)} \boldsymbol{C} Q,
\end{aligned}
$$

where

$$
C_{i j}=\frac{\partial^{2} c}{\partial n_{i} \partial n_{j}},
$$

i.e., the elements of $\boldsymbol{C}$ correspond to second order derivatives of sound speed along either $\boldsymbol{e}_{1}, \boldsymbol{e}_{2}$, or both. Generally speaking $\boldsymbol{P}$ describes the beam slowness in the plane perpendicular to $\boldsymbol{e}_{s}$, while $\boldsymbol{Q}$ describes the beam spreading. The pair of expressions given by Eq. (6) is called the dynamic equations of the full Gaussian beam formulation. The expression given by Eq. (1) behaves near the source like an spherical wave emitted by a point source through the choice of initial conditions $^{19}$

$$
\boldsymbol{P}(0)=\left[\begin{array}{cc}
1 & 0 \\
0 & \cos \theta(0)
\end{array}\right] / c(0)
$$

and $^{17}$

$$
\boldsymbol{Q}(0)=\left[\begin{array}{ll}
0 & 0 \\
0 & 0
\end{array}\right]
$$

Generally speaking the full Gaussian beam approach is difficult to implement numerically, with the main difficulties being related to refraction effects (the problem of ray boundary reflection is in fact much easier to account for). In particular, when horizontal refraction is considered, rays with a common initial azimuth exhibit ray trajectories which do not lie on a common plane; besides, horizontal refraction also leads to the rotation of polarization vectors along a given ray trajectory, inducing a large variability of beam shapes within any group of rays (even if the initial orientation of polarization vectors was the same for all rays). Additionally, the calculation of beam influence (which requires a proper calculation of the matrix $\boldsymbol{C}$ ) for the arbitrary position of an hydrophone is cumbersome. Other issues related to the calculation of eigenrays, such as determining arrivals and respective amplitudes, are also difficult to implement. In order to develop a two-dimensional application of Eq. (1), TRACEO relies on the particular solution of dynamic and Eikonal equations when horizontal refraction is absent. For such case there is no rotation of the polarization vectors; thus, choosing $\boldsymbol{e}_{2}$ to lie on the horizontal plane fixes the positioning of $\boldsymbol{e}_{1}$ on a plane of constant azimuth (perpendicular to $\boldsymbol{e}_{2}$ ). Beam amplitude is then calculated by TRACEO on the plane of constant azimuth by considering the particular solution with $n_{2}=0$. Since the particular solution does not exhibit cylindrical symmetry the approximation used by TRACEO can be regarded as a Gaussian beam solution on the $(x, z)$ plane (i.e., on the plane corresponding to azimuth zero).

Under the conditions above considered (i.e., absence of horizontal refraction and $\boldsymbol{e}_{2}$ placed initially on the horizontal plane) one can write that

$$
\boldsymbol{C}=\left[\begin{array}{cc}
c_{11} & 0 \\
0 & 0
\end{array}\right]
$$

Without loss of generality the matrix $\boldsymbol{Q}$ can be represented as

$$
\boldsymbol{Q}(s)=\left[\begin{array}{cc}
q(s) & 0 \\
0 & q_{\perp}(s)
\end{array}\right]
$$

so $\operatorname{det} \boldsymbol{Q}=q(s) q_{\perp}(s)$. Combining the initial conditions for a point source with the given approximations one can substitute the pair Eq. (6) with the expressions

$$
\frac{d}{d s} q=c(s), \quad \frac{d}{d s} p=-\frac{c_{11}}{c(s)^{2}} q,
$$

where $p=p_{11} \quad$ it can be shown that $p_{12}=p_{21}=0$, $\left.p_{22}=\cos \theta(0) / c(0)\right]$; thus, the particular solution of Eq. (1) can be written as

$$
\begin{aligned}
P\left(s, n_{1}, n_{2}\right)= & \frac{1}{4 \pi} \sqrt{\frac{c(s)}{c(0)} \frac{\cos \theta(0)}{q_{\perp}(s) q(s)}} \\
& \times \exp \left[-i \omega\left(\tau(s)+\frac{1}{2} \frac{p(s)}{q(s)} n_{1}^{2}+\frac{1}{2 I_{c}(s)} n_{2}^{2}\right)\right],
\end{aligned}
$$


where

$$
I_{c}(s)=\int_{0}^{s} c\left(s^{\prime}\right) d s^{\prime}
$$

and

$$
q_{\perp}(s)=\frac{\cos \theta(0)}{c(0)} I_{c}(s) .
$$

Taking $n_{2}=0$ in Eq. (13) and representing $n_{1}$ simply as $n$ one can write the solution on the plane of constant azimuth as

$$
\begin{aligned}
P(s, n)= & \frac{1}{4 \pi} \sqrt{\frac{c(s)}{c(0)} \frac{\cos \theta(0)}{q_{\perp}(s) q(s)}} \\
& \times \exp \left[-i \omega\left(\tau(s)+\frac{1}{2} \frac{p(s)}{q(s)} n^{2}\right)\right] .
\end{aligned}
$$

Equation (16) is similar to the Gaussian beam expression for a waveguide with cylindrical symmetry ${ }^{18,20}$

$$
P(s, n)=\frac{1}{4 \pi} \sqrt{\frac{c(s)}{c(0)} \frac{\cos \theta(0)}{r q(s)}} \exp \left[-i \omega\left(\tau(s)+\frac{1 p(s)}{2 q(s)} n^{2}\right)\right] .
$$

The term $1 / \sqrt{r}$ appears in Eq. (17) due to the cylindrical spreading of the pressure field, and the expression itself is an asymptotic solution of the wave equation, which breaks down at the source position. Therefore, a "blind" numerical application of Eq. (17) to rays propagating back to the source produces waves, which are focused back to the source and break down in its vicinity. When compared to Eq. (17) one can notice that Eq. (16) contains the parameter $q_{\perp}(s)$ instead of $r$; since $q_{\perp}(s)$ is proportional to the ray arclength, beam amplitudes given by Eq. (16) always decrease independently of rays propagating forwards or backwards. This feature of Eq. (16) is expected to be relevant for backscattering studies.

The field given by Eq. (16) is not sufficient for TRACEO to account properly for phase and amplitude corrections every time a ray hits a boundary; in such case the beam amplitude is multiplied by a boundary reflection coefficient, which takes into account shear speed and shear attenuation; ${ }^{21}$ the full expression of such reflection coefficient is presented in Appendix A. Additional corrections to the ray amplitude are introduced using finite element ray tracing ${ }^{22}$ and phase corrections induced by caustics (which are described in detail in Ref. 18).

To understand the method implemented in TRACEO for particle velocity calculations let us recall that particle velocity $v$ is related to acoustic pressure $P$ in the frequency domain through the relationship ${ }^{23}$

$$
\boldsymbol{v}=-\frac{i}{\omega \rho} \nabla P
$$

where $\rho$ represents the watercolumn density, and $\omega$ stands for the frequency of the propagating wave. The factors $\rho$ and $\omega$ only affect the amplitude of $\boldsymbol{v}$, while the imaginary unit implies a phase shift of $\pi / 2$ radians. Without these factors particle velocity can be viewed as the gradient of acoustic pressure. To obtain this gradient, TRACEO calculates the acoustic pressure on a star-shaped stencil, with the hydrophone located at the star's center; outer points are located at the coordinates $(r \pm \Delta, z \pm \Delta)$. The points aligned along the horizontal are used to calculate the coefficients of a parabolic interpolator (described in Appendix B), and those coefficients allow determination of the horizontal derivative at the center; a similar procedure is followed for the points aligned along the vertical. To avoid aliasing, the spacing between an outer point and the center is taken (arbitrarily) as corresponding to $\Delta=\lambda / 10$, where $\lambda$ represents the acoustic wavelength. Interpolation is preferred to analytic expressions derived from Eq. (16) or Eq. (17) because either of them is written in terms of ray coordinates $(s, n)$, instead of horizontal and vertical coordinates. Such analytic expressions are elaborate and can be used only with a Gaussian beam model, while the interpolation approach is valid for any model and can easily be extended to three dimensions.

\section{B. Numerical example}

The capabilities of TRACEO require intense testing through comparisons with other models, a discussion of backscattering issues in more detail and comparison between experimental data and field predictions when targets are present in the water column (just to mention a few further directions of research). Such issues go far beyond the main goals of the discussion presented here and will be addressed in future studies. The numerical example in this section is limited to a comparison of TRACEO predictions of the horizontal and vertical components of particle velocity (hereafter represented as $u$ and $w$, respectively), with the corresponding values found from analytic expressions for an elastic bottom Pekeris waveguide (shear included); the compressional and shear potentials of such a waveguide are well known in the literature, ${ }^{24}$ and the particle velocity components are easily calculated from the analytic expressions for both potentials. It is worth remarking that, from the point of view of normal modes, the contribution of lower-order modes is enhanced in the field of $u$, while the field of $w$ is enhanced by the contribution of higher-order modes. ${ }^{25}$ The comparison between particle velocity calculations from the analytic solution and TRACEO predictions for the flat waveguide $(200 \mathrm{~Hz}$, $z_{0}=z_{1}=145 \mathrm{~m}$ ) is shown in Fig. 2; in both cases the analytic solution is indicated by the solid curve, while the model prediction is indicated by the dashed curve. The interpolation approach is so accurate in the case of $u$ that the two curves are difficult to distinguish [Fig. 2(a)]. The approach is less accurate for $w$, with the model exhibiting some overshooting of the analytic solution [Fig. 2(b)], although it does correctly reproduce the interference pattern in both phase and amplitude in range.

\section{BENCHMARKING}

Seismo-acoustic benchmarking of the ray solution against tank data is discussed in this section through a 

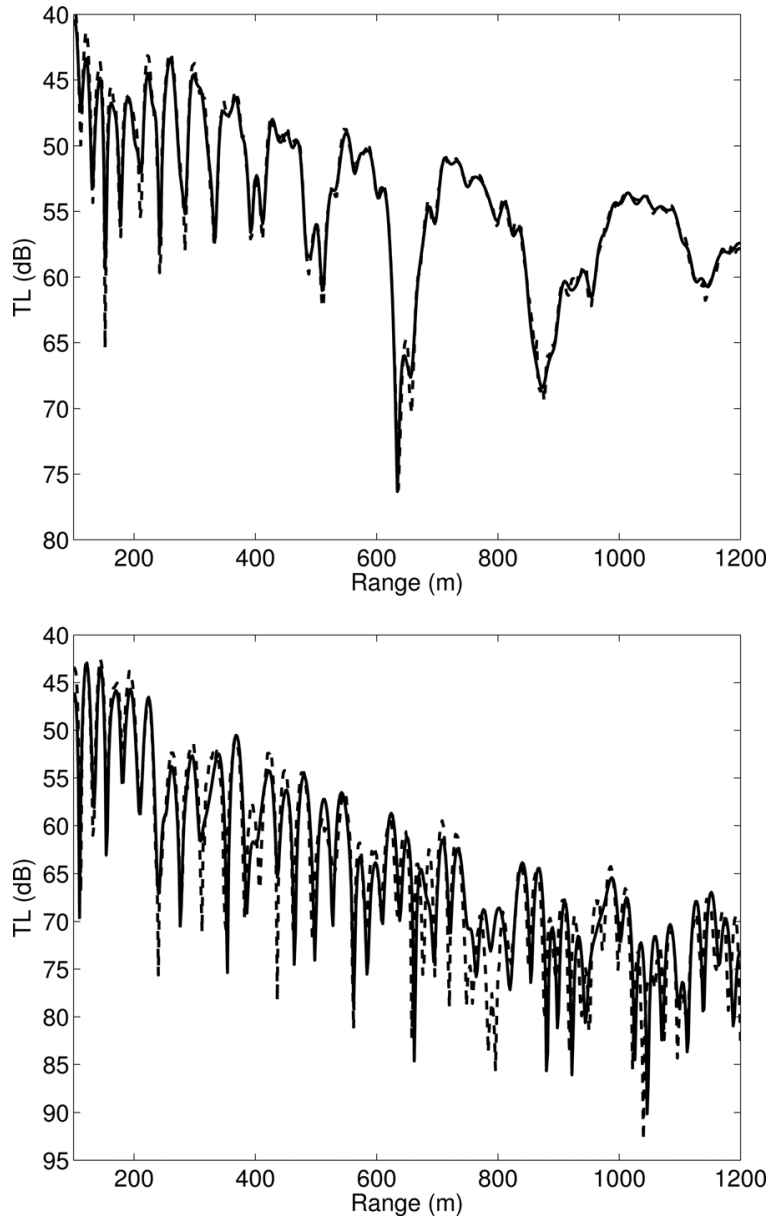

FIG. 2. Particle velocity calculations at $200 \mathrm{~Hz}$ for the flat waveguide $\left(z_{0}=z_{1}=145 \mathrm{~m}\right): u$ (top); $w$ (bottom). Solid curve: analytic solution; dashed curve: TRACEO's prediction (compare with the middle plot of Fig. 3).

systematic set of comparisons with transmission loss curves calculated from tank experimental data at model frequencies of 125, 200, and $275 \mathrm{~Hz}$. Comparisons against EPEE-1 data are discussed in Secs. IV A and IV B for the flat and upslope waveguides, while comparisons againts EPEE-2 data for the flat to downslope, upslope to flat, and upslope to downslope waveguides are discussed in Secs. IV C-IVE. Results from Secs. IV A and IV B can be related to Figs. 4 and 7 from Ref. 6, respectively; the results for the EPEE-2 data concern source-receiver configurations not discussed in Ref. 7. In all sections, tank data is shown in the figures as a solid curve, while the dashed curve indicates TRACEO predictions; additionally, the plots are arranged with frequency increasing from top to bottom; geometries (source depth, receiver depth, and source-receiver range) are all given in model values. It is worth remarking that the benchmarking was not limited to the mentioned set of frequencies. Additional comparisons were performed at model frequencies of 100 and $300 \mathrm{~Hz}$, with no appreciable deviation from what was found at the chosen set of frequencies. Since no new information was provided by the benchmarking at such frequencies, comparisons are not included in the discussion. In order to produce TRACEO predictions as objectively as possible the following procedure was followed: the number of rays was taken, arbitrarily, as high as 201 rays, in order to ensure that field coherence was properly modeled at all frequencies. Source aperture corresponded to $55.25^{\circ}$; that value was obtained by minimizing the standard deviation over apertures in the interval $\left[35^{\circ}, 85^{\circ}\right]$ of the difference between the experimental transmission loss and the model transmission loss for the flat waveguide at the "central" model frequency of $200 \mathrm{~Hz}$. Thus, 201 rays between $-55.25^{\circ}$ and $55.25^{\circ}$ were calculated by TRACEO at all frequencies, for all waveguides, and for all considered source/receiver configurations.
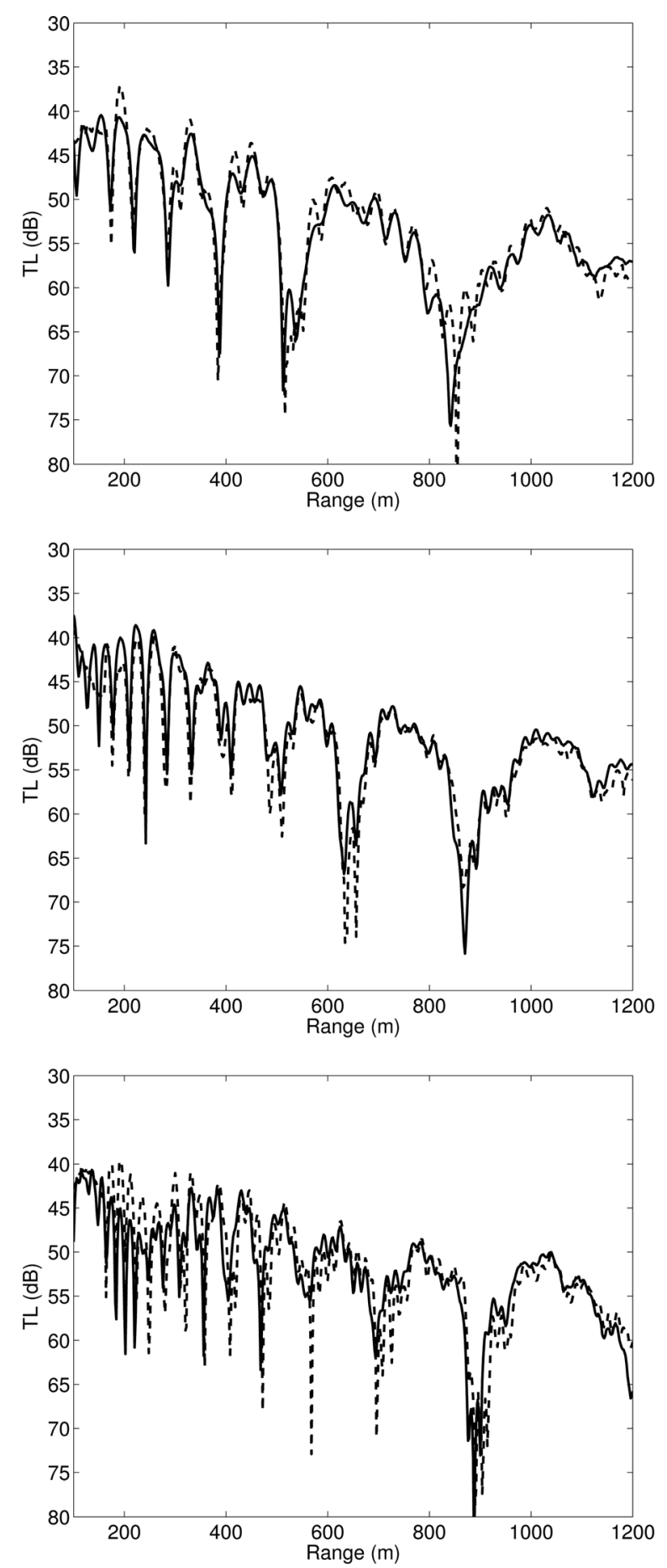

FIG. 3. Benchmarking for the flat waveguide, source at $69.1 \mathrm{~m}$ and receiver at $137.1 \mathrm{~m}: 125 \mathrm{~Hz}$ (top); $200 \mathrm{~Hz}$ (middle); $275 \mathrm{~Hz}$ (bottom). Solid curve: tank data; dashed curve: TRACEO's prediction (compare with Fig. 4 of Ref. 6). 


\section{A. Flat waveguide}

Tank experiment and model curve comparisons for the flat waveguide are shown in Fig. 3. In all cases the ray model consistently and accurately reproduces the phase and amplitude behavior of experimental data, although occasional overshooting is observed. Despite the eventual limitations that one would expect from ray predictions at low frequencies the match between TRACEO and experimental data is remarkably accurate at $125 \mathrm{~Hz}$. The results can be compared
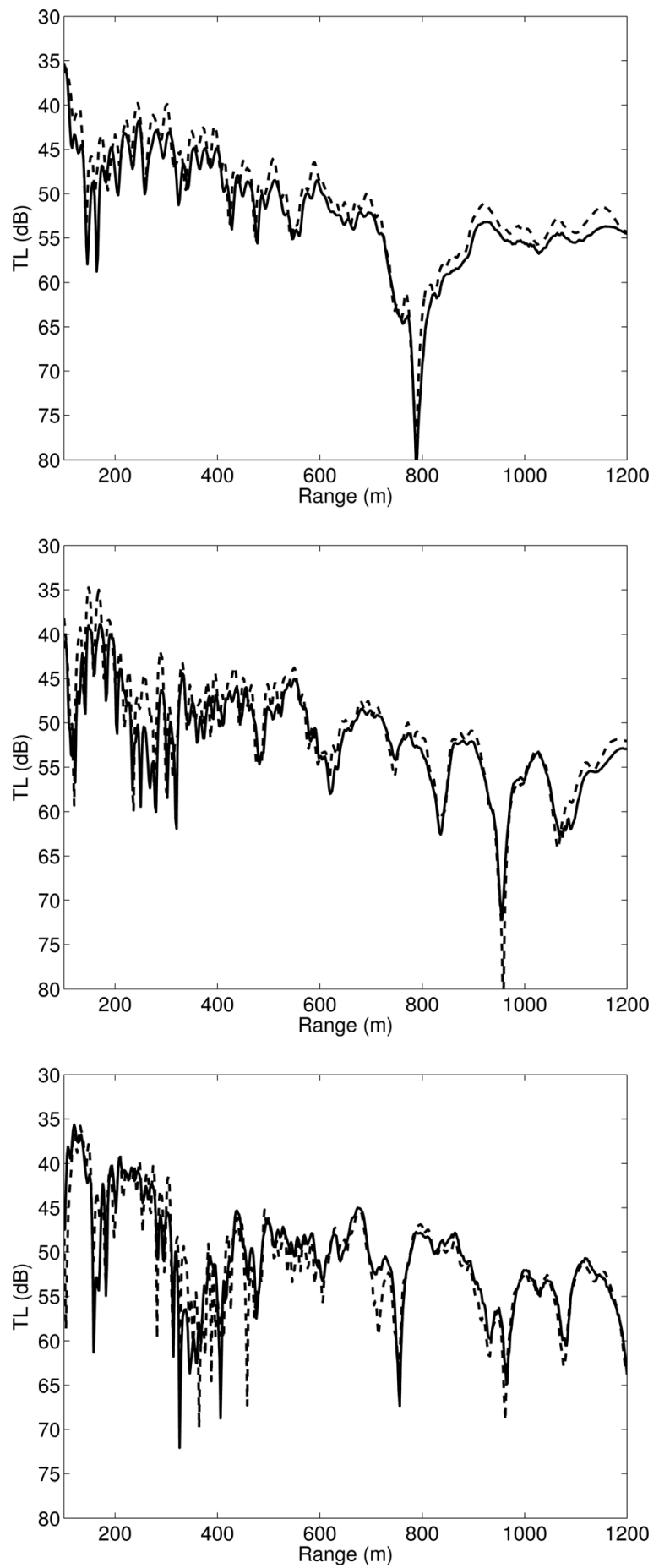

FIG. 4. Benchmarking for the upslope waveguide, source at $63.4 \mathrm{~m}$ and receiver at $15.6 \mathrm{~m}: 125 \mathrm{~Hz}$ (top); $200 \mathrm{~Hz}$ (middle); $275 \mathrm{~Hz}$ (bottom). Solid curve: tank data; dashed curve: TRACEO's prediction (compare with Fig. 7 of Ref. 6). directly with Fig. 4 of Ref. 6 and indicate that for the given configuration the accuracy of both TRACEO and ROTVARS is nearly the same.

\section{B. Upslope waveguide}

Tank experiment data and model curve comparisons for the upslope waveguide are shown in Fig. 4. As one might
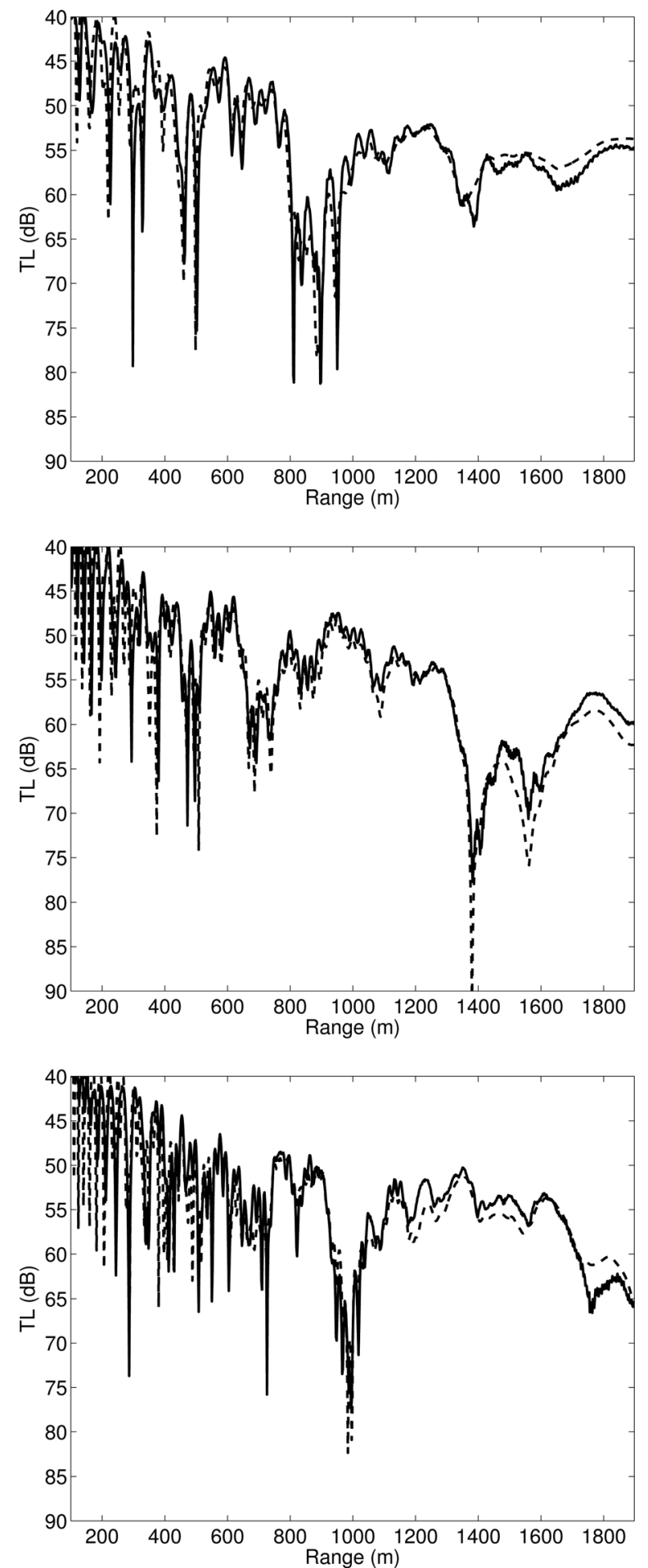

FIG. 5. Benchmarking for the flat to downslope waveguide, source at $74.2 \mathrm{~m}$ and receiver at $75.8 \mathrm{~m}: 125 \mathrm{~Hz}$ (top); $200 \mathrm{~Hz}$ (middle); $275 \mathrm{~Hz}$ (bottom). Solid curve: tank data; dashed curve: TRACEO's prediction (compare with Figs. 2 and 3 of Ref. 7).

Camargo Rodríguez et al:: Ray model benchmarking against tank data 
expect ray results improve as frequency increases and TRACEO produces accurate predictions in all cases (with matches that at first glance appear even more accurate than those of the flat waveguide). The match is surprisingly good in many cases near the final ranges if one takes into account the existing bottom gap beyond the end of the PVC slab. The results can be compared directly with Fig. 7 of Ref. 6 and indicate, one more time, that for the given configuration
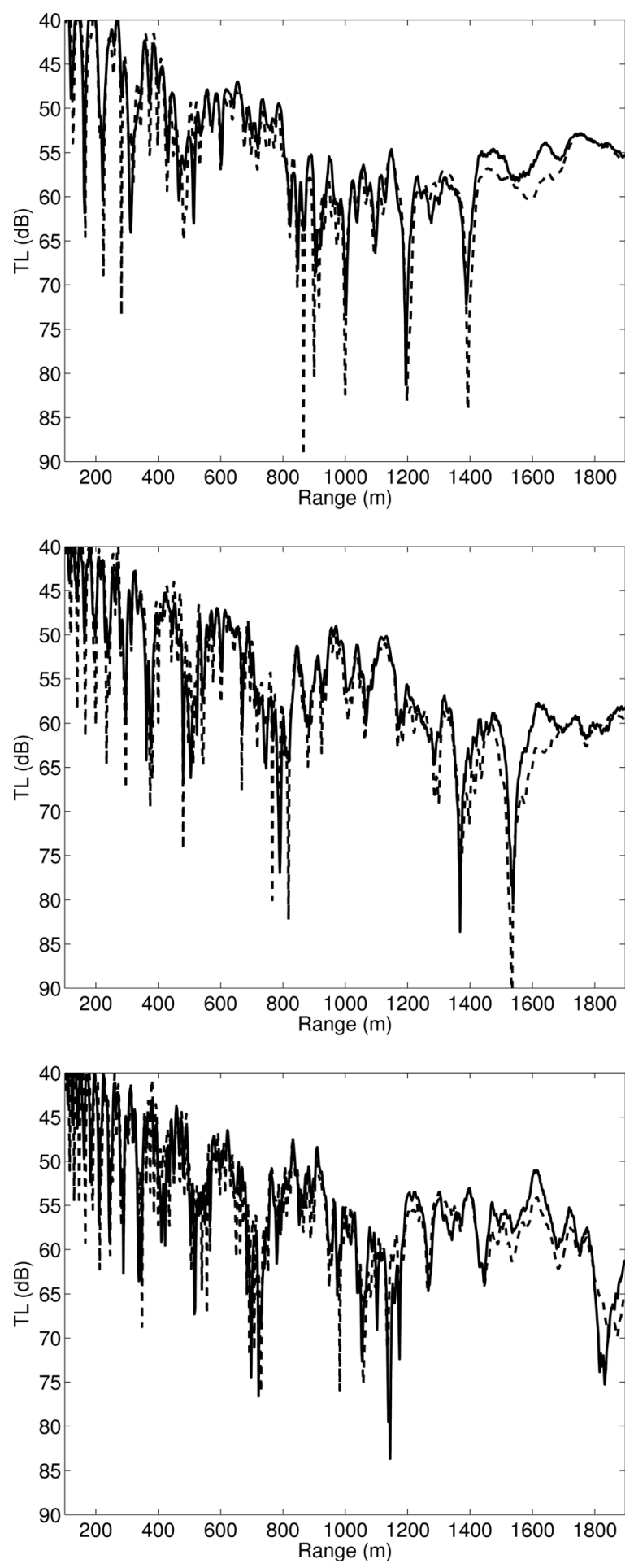

FIG. 6. Benchmarking for the upslope to flat waveguide, source at $73.5 \mathrm{~m}$ and receiver at $78.7 \mathrm{~m}: 125 \mathrm{~Hz}$ (top); $200 \mathrm{~Hz}$ (middle); $275 \mathrm{~Hz}$ (bottom). Solid curve: tank data; dashed curve: TRACEO's prediction (compare with Figs. 4 and 5 of Ref. 7). there are no significant differences in the predictions produced by either TRACEO Or ROTVARS.

\section{Flat to downslope waveguide}

Tank experiment data and model curve comparisons for the flat to dowslope waveguide are shown in Fig. 5. The figures reveal some undershooting or overshooting of the
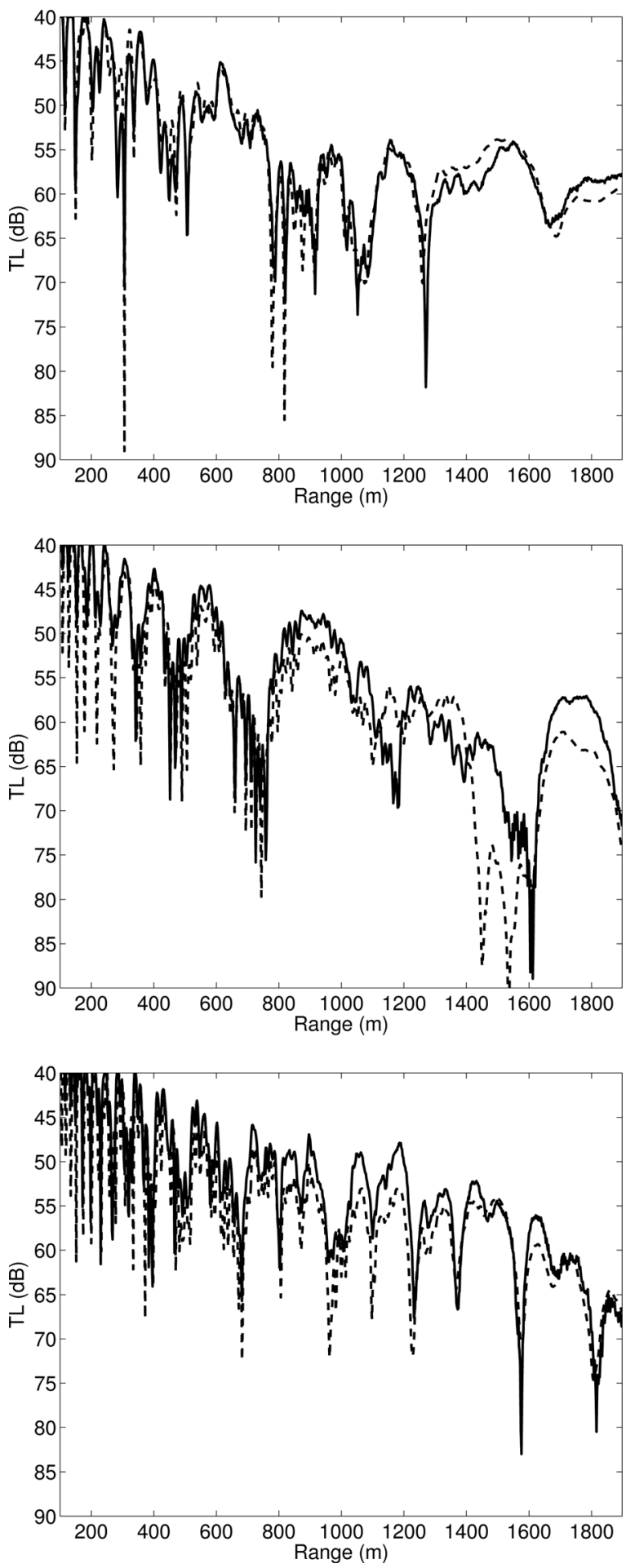

FIG. 7. Benchmarking for the upslope to downslope waveguide, source at $72.1 \mathrm{~m}$ and receiver at $74.8 \mathrm{~m}: 125 \mathrm{~Hz}$ (top); $200 \mathrm{~Hz}$ (middle); $275 \mathrm{~Hz}$ (bottom). Solid curve: tank data; dashed curve: TRACEO's prediction (compare with Fig. 6 of Ref. 7). 
solution near the end of the slab, although the behavior is not consistent over frequency. Curiously the slight deviation of TRACEO's prediction from experimental data does not start at the range where the slab ends, but slightly beyond that range. Despite the slight mismatch, TRACEO properly reproduces the phase variations of the experimental data. A partial comparison can be done with Figs. 2 and 3 from Ref. 7 (the data is from the EPEE-2 experiment, but for different source and receiver depths), which indicates that TRACEO exhibits nearly the same accuracy as ROTVARs.

\section{Upslope to flat waveguide}

Tank experiment data and model curve comparisons for the upslope to flat waveguide are shown in Fig. 6. In this case the general trend of the ray model is to slightly undershoot the experimental data near the final ranges, although phase variations are again accurately reproduced by the numerical solution. A partial comparison can be done with Figs. 4 and 5 from Ref. 7 (the data are from the EPEE-2 experiment, but for different source and receiver depths), which shows no significant differences in the accuracy of either TRACEO Or ROTVARS.

\section{E. Upslope to downslope waveguide}

Tank experiment and model curves for the upslope to downslope waveguide are shown in Fig. 7. This case could be considered to be the most difficult to simulate because of the significant change in bottom slope and it reveals in fact a set of less satisfactory matches to the experimental data. The comparisons are somehow intriguing for two reasons: first, one can notice that the mismatch is much more severe at the "central" model frequency of $200 \mathrm{~Hz}$; second, the mismatch at $275 \mathrm{~Hz}$ is more severe near the middle of the waveguide than near the end. Additional comparisons with the RAMS model $^{26}$ (not shown here) produced a similar set of results. Curiously, Fig. 6 in Ref. 7 (which is related also to the EPEE-2 experiment, but for different source and receiver depths) exhibits a similar pattern.

\section{CONCLUSIONS AND FUTURE WORK}

The discussion presented in the previous sections demonstrated the feasibility of using a ray approach for seismoacoustic studies related to acoustic propagation over isotropic elastic bottoms. Systematic benchmarking of the TRACEO ray model to experimental tank data, representative of propagation over elastic bottoms with sharp slope transitions exhibited a high degree of accuracy, comparable to the one already found with ROTVARS. As an experimental validation of the TRACEO model the results are extremely encouraging for further model applications, given the unique model features. Such applications can be oriented, for instance, to seismoacoustic inversion of both compressional and shear bottom properties, using either standard hydrophone or vector sensor arrays, as long as the bottom does not exhibit a complex layered structure. Benchmarking results shown in Sec. IV indicate that such applications do not require necessarily to deal with high frequency propagation, since TRACEO exhibited a high accuracy at relatively low frequencies. A preliminary comparison of computational times between TRACEO and RAMS (which is widely available) on a typical laptop produced average values of $0.9 \mathrm{~s}$ vs $1.8 \mathrm{~s}$, respectively, for the configurations of the EPEE-1 experiment, and of $1.8 \mathrm{~s}$ vs $5.2 \mathrm{~s}$, respectively, for the configurations of the EPEE-2 experiment. Thus this particular trend shows TRACEO being faster than RAMS, although model parameters in the two cases were not optimized to minimize computational time without compromising accuracy. At high frequencies differences in computational times can be expected to become more relevant. Future directions of research will necessarily include detailed comparisons with field data (where mismatch can be expected to become more relevant), studies of backscattering issues (based on benchmarking against analytic solutions, backscattering-capable models, and/or available experimental data), accounting for ray tracing in elastic layered systems and three-dimensional field predictions where a ray approach looks like an attractive alternative for efficient and fast field computations.

\section{ACKNOWLEDGMENTS}

The authors are deeply thankful to the Naval Research Laboratory (NRL) for allowing the use of the experimental data discussed in this work. This work was supported by the Portuguese Foundation for Science and Technology through the SENSOCEAN project (FCT Contract No. PTDC/EEAELC/104561/2008).

\section{APPENDIX A: RAY MODEL REFLECTION COEFFICIENT FOR AN ELASTIC BOTTOM}

The calculation of the reflection coefficient for the elastic bottom is given by the following expression: ${ }^{21}$

$$
R\left(\theta_{1}\right)=\frac{D\left(\theta_{1}\right) \cos \theta_{1}-1}{D\left(\theta_{1}\right) \cos \theta_{1}+1},
$$

where

$$
\begin{aligned}
& D\left(\theta_{1}\right)=A_{1}\left(A_{2}\left(1-A_{7}\right) / \sqrt{1-A_{6}^{2}}\right. \\
& \left.+A_{3} A_{7} / \sqrt{1-A_{5} / 2}\right) \\
& A_{1}=\frac{\rho_{2}}{\rho_{1}}, \quad A_{2}=\frac{\tilde{c}_{p 2}}{c_{p 1}}, \quad A_{3}=\frac{\tilde{c}_{s 2}}{c_{p 1}}, \\
& A_{4}=A_{3} \sin \theta_{1}, \quad A_{5}=2 A_{4}^{2}, \quad A_{6}=A_{2} \sin \theta_{1} \text {, } \\
& A_{7}=2 A_{5}-A_{5}^{2} \text {, } \\
& \tilde{c}_{p 2}=c_{p 2} \frac{1-i \tilde{\alpha}_{c p}}{1+\tilde{\alpha}_{c p}^{2}}, \quad \tilde{c}_{s 2}=c_{s 2} \frac{1-i \tilde{\alpha}_{c s}}{1+\tilde{\alpha}_{c s}^{2}}, \\
& \tilde{\alpha}_{c p}=\frac{\alpha_{c p}}{40 \pi \log e}, \quad \tilde{\alpha}_{c s}=\frac{\alpha_{c s}}{40 \pi \log e} ;
\end{aligned}
$$

the units of attenuation should be given in $\mathrm{dB} / \lambda$ and the angle $\theta_{1}$ is given relative to normal to the bottom (see Fig. 8). In general the reflection coefficient is real when $\alpha_{c p}=\alpha_{c s}=0$, and the angle of incidence $\theta_{1}$ is less than the critical angle $\theta_{c r}$, with $\theta_{c r}$ given by the expression 


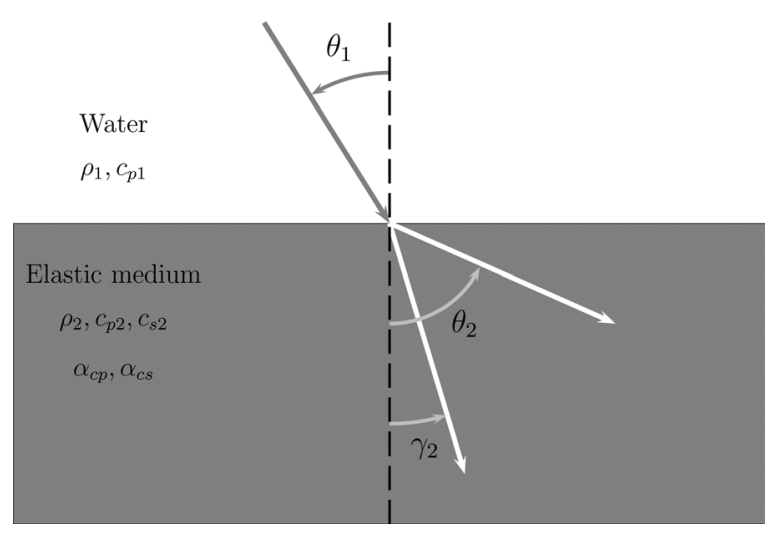

FIG. 8. Ray reflection at an elastic media interface.

$$
\theta_{c r}=\arcsin \left(\frac{c_{p 1}}{c_{p 2}}\right)
$$

Attenuation can be expected to be negligible when $\theta_{1}<\theta_{c r}$, and for small $\theta_{1}$ the energy transferred to shear waves in the elastic medium is only a small fraction of the total energy transferred.

\section{APPENDIX B: BARYCENTRIC PARABOLIC INTERPOLATION}

The barycentric parabolic interpolator can be described as follows: let us consider a set of three points $x_{1}, x_{2}$, and $x_{3}$ aligned along the $X$ axis, and the corresponding function values $f\left(x_{1}\right), f\left(x_{2}\right)$, and $f\left(x_{3}\right)$. At a given point $x$ between $x_{1}$ and $x_{3}$ the interpolant can be written as

$$
f(x)=f\left(x_{1}\right)+a_{2}\left(x-x_{1}\right)\left(x-x_{3}\right)+a_{3}\left(x-x_{1}\right)\left(x-x_{2}\right) .
$$

It follows from this expression that

$$
a_{2}=\frac{f\left(x_{2}\right)-f\left(x_{1}\right)}{\left(x_{2}-x_{1}\right)\left(x_{2}-x_{3}\right)} \text { and } a_{3}=\frac{f\left(x_{3}\right)-f\left(x_{1}\right)}{\left(x_{3}-x_{1}\right)\left(x_{3}-x_{2}\right)} .
$$

The approximations for the derivatives become

$$
\frac{d f}{d x}=a_{2}\left(2 x-x_{1}-x_{3}\right)+a_{3}\left(2 x-x_{1}-x_{2}\right)
$$

and

$$
\frac{d^{2} f}{d x^{2}}=2\left(a_{2}+a_{3}\right)
$$

${ }^{1}$ F. Jensen and C. Ferla, "Numerical solution of range-dependent benchmark problems in ocean acoustics," J. Acoust. Soc. Am. 87, 1499-1510 (1990).
${ }^{2}$ R. Stephen, "Solutions to range-dependent benchmark problems by the finite-difference method," J. Acoust. Soc. Am. 87, 1527-1534 (1990).

${ }^{3}$ D. Thomson and G. Brooke, "Numerical implementation of a modal solution to a range-dependent benchmark problem," J. Acoust. Soc. Am. 87, $1521-1526$ (1990).

${ }^{4}$ E. Westwood, "Ray model solutions to the benchmark wedge problems," J. Acoust. Soc. Am. 87, 1539-1545 (1990).

${ }^{5} \mathrm{M}$. Buckingham and A. Tolstoy, "An analytical solution for benchmark problem 1: The ideal wedge," J. Acoust. Soc. Am. 87, 1511-1513 (1990).

${ }^{6}$ J. Collis, W. Siegmann, M. Collins, H. Simpson, and R. Soukup, "Comparison of simulations and data from a seismo-acoustic tank experiment," J. Acoust. Soc. Am. 122, 1987-1993 (2007).

${ }^{7}$ H. Simpson, J. Collis, R. Soukup, M. Collins, and W. Siegmann, "Experimental testing of the variable rotated elastic parabolic equation," J. Acoust. Soc. Am. 130, 2681-2686 (2011).

${ }^{8}$ D. Outing, W. Siegmann, M. Collins, and E. Westwood, "Generalization of the rotated parabolic equation to variable slopes," J. Acoust. Soc. Am. 6, 3534-3538 (2006).

${ }^{9} \mathrm{M}$. Porter, The BELLHOP Manual and User's Guide (HLS Research, La Jolla, CA, 2011).

${ }^{10}$ O. Rodríguez, "General description of the BELLHOP ray tracing model," technical report, 2008 (Last viewed 06/15/2012).

${ }^{11}$ E. Westwood and P. Vidmar, "Eigenray finding and time series simulation in a layered-bottom ocean," J. Acoust. Soc. Am. 81, 912-924 (1987).

${ }^{12} \mathrm{H}$. Weinberg and R. Keenan, "Gaussian ray bundles for modeling highfrequency propagation loss under shallow-water conditions," J. Acoust. Soc. Am. 100, 1421-1431 (1996).

${ }^{13} \mathrm{http}: / /$ www.siplab.fct.ualg.pt (Last viewed 06/15/2012).

${ }^{14}$ P. Santos, P. Felisberto, O. Rodríguez, and S. Jesus, "Geoacoustic matched-field inversion using a vector sensor array," in Conference on Underwater Acoustic Measurements, Nafplion, Greece (2009), pp. 29-34.

${ }^{15}$ P. Santos, O. Rodríguez, P. Felisberto, and S. Jesus, "Seabed geoacoustic characterization with a vector sensor array," J. Acoust. Soc. Am. 128, 2652-2663 (2010).

${ }^{16}$ P. Santos, P. Felisberto, O. Rodríguez, J. Joao, and S. Jesus, "Geometric and Seabed parameter estimation using a Vector Sensor Array-Experimental results from Makai experiment 2005," in OCEANS2011, Santander, Spain (2011), pp. 1-10.

${ }^{17}$ M. Popov and I. Peník, "Computation of ray amplitudes in homogeneous media with curved interfaces," Studia Geoph. Geod. 22, 248-258 (1978).

${ }^{18}$ F. Jensen, W. Kuperman, M. Porter, and H. Schmidt, Computational Ocean Acoustics, AIP Series in Modern Acoustics and Signal Processing (AIP, New York, 1994), Chap. 3.

${ }^{19}$ M. Popov, "Ray theory and Gaussian beam method for geophysicists," technical report EDUFBA, Universidade Federal da Bahia (2002).

${ }^{20} \mathrm{M}$. Porter and H. Bucker, "Gaussian beam tracing for computing ocean acoustic fields," J. Acoust. Soc. Am. 82, 1349-1359 (1987).

${ }^{21}$ P. Papadakis, M. Taroudakis, and J. Papadakis, "Recovery of the properties of an elastic bottom using reflection coefficient measurements," in Proceedings of the 2nd European Conference on Underwater Acoustics, Copenhagen, Denmark (1994), Vol. II, pp. 943-948.

${ }^{22}$ M. Porter and Y.-C. Liu, "Finite-Element Ray Tracing," in Theoretical and Computational Acoustics (World Scientific Publishing, Singapore, 1994), Vol. 2, pp. 947-956.

${ }^{23} \mathrm{H}$. Schmidt, "SAFARI, seismo-acoustic fast field algorithm for rangeindependent enviroments. User's guide," technical report, Saclant Undersea Research Centre (SM-113), La Spezia, Italy (1987).

${ }^{24} \mathrm{~F}$. Press and M. Ewing, "Propagation of explosive sound in a liquid layer overlying a semi-infinite elastic solid," Geophysics 15, 426-446 (1950).

${ }^{25}$ P. Felisberto, O. Rodríguez, P. Santos, and S. Jesus, "On the usage of the particle velocity field for bottom characterization," in International Conference on Underwater Acoustic Measurements, Kos, Greece (2011), pp. $19-24$.

${ }^{26} \mathrm{M}$. Collins, User's Guide for RAM Versions 1.0 and 1.0p (Naval Research Laboratory, Washington, DC, 2006). 